\title{
Bedside nuclear probe for detection and quantification of left to right intracardiac shunts
}

\author{
BRIAN A GOULD, JOHN TURNER, DAVID H KEELING, NICHOLAS J RING, \\ ROBERT R COX, ANDREW J MARSHALL \\ From the Departments of Cardiology, Nuclear Medicine, and Radiology, Plymouth Group of Hospitals, \\ Plymouth
}

SUMMARY A cadmium telluride nuclear probe with an Elscint gamma camera was used to detect and measure left to right intracardiac shunts at the bedside in 34 patients. Fifteen also had right heart catheterisation and oximetric measurement of the shunt. For the nuclear technique $740 \mathrm{MBq}$ $(20 \mathrm{mCi})$ of technetium-99m pertechnetate was injected into the right antecubital vein and the pulmonary to systemic flow ratio (QP:QS) was measured by the gamma variate technique. Data were not obtained in four patients because the nuclear probe failed in three patients and one storage disc was corrupted. Data from the gamma camera were lost in another patient. When the size of the shunt measured by the nuclear probe was compared with that measured by the oximetric technique the mean difference (SD of mean difference) was 0.36 (SD 0.78) and when it was compared with the gamma camera it was 0.08 (SD 0.67). Analysis of scatter plots showed that the larger the shunt, the larger the discrepancy. Twenty four of 29 data sets showed complete agreement between the nuclear probe and gamma camera on the size of the shunt. Any differences were small.

These data indicate that left to right intracardiac shunts may be measured accurately by a nuclear probe at the bedside in either the coronary care unit or outpatient department.

A method for detecting and measuring left to right intracardiac shunts at the bedside would be useful. It could rapidly confirm the clinical diagnosis of suspected rupture of the interventricular septum in outpatients and in the coronary care unit.

Earlier studies have shown good agreement between the results of a radionuclide technique and oximetry $^{1-3}$ and some believe that the radionuclide technique is more accurate. ${ }^{4}$ We have compared the estimates of intracardiac shunts measured by a gamma camera and by a miniaturised cadmium telluride nuclear probe with those of standard oximetry.

\section{Patients and methods}

To test the discriminatory function of the radionuclide technique we studied 28 patients (mean (range) age $38(14-72)$ ) with suspected left to right

Requests for reprints to Dr Andrew J Marshall, Greenbank Hospital, Plymouth PL4 8NN.

Accepted for publication 29 October 1987 shunts and a further six with either impaired left ventricular function or mitral regurgitation. Fifteen patients in whom clinically significant shunts were suspected also had right heart catheterisation and oximetric measurement of the shunt.

Two experienced consultant radiologists independently reported on the size of the shunts from standard chest $x$ rays and graded the size of the shunt $(\mathrm{A}=$ no shunt; $\mathrm{B}=\mathrm{QP}: \mathrm{QS}<2: 1$; and $\mathrm{C}=\mathrm{QP}: \mathrm{QS}$ $>2: 1)$.

\section{RADIONUCLIDE TECHNIQUE}

A first pass radionuclide angiogram was acquired in the anterior projection simultaneously for both the gamma camera and nuclear probe. Data were collected in list mode by an Elscint gamma camera linked to a PDP 11 computer. Two miniaturised cadmium telluride detectors were positioned on the anterior chest wall and the patient was positioned under the gamma camera. One nuclear probe was located over the clavicular region to indicate the compactness of the bolus at the' superior vena cava and the other was positioned over the right lung to detect a left to right shunt, which is shown by a prolongation of the normal time activity curve. During the study we 
found it necessary to provide additional collimation for these probes to prevent pick up of activity in adjacent areas (for example the superior vena cava and aorta).

These probes were connected to a solid state memory recorder (Memolog - 500, Novo Diagnostics). The data were later transferred to the PDP 11 computer, which was used to calculate the size of the shunt. The patient's red cells were prepared for in vivo labelling by a preliminary intravenous injection of (inactive) pyrophosphate and stannous ion. Twenty minutes later, $740 \mathrm{MBq}(20 \mathrm{mCi})$ of technetium-99m pertechnetate was injected into the right antecubital vein. The nuclear probe would require $150 \mathrm{MBq}(4 \mathrm{mCi})$ of technetium-99m when used alone.

Early in the study the bolus was injected through a $18 \mathrm{G}$ Venflon. Because some of the injections gave a fractionated bolus the injection technique was modified. The Venflon was connected to a short length of clear tubing which was connected to a three-way tap. A $1 \mathrm{ml}$ syringe containing $99 \mathrm{~m}$ Tc was connected to the side arm and a $50 \mathrm{ml}$ syringe of saline was connected to the other. The three-way tap and tubing were loaded with saline. The dose of technetium, marked on both sides by a small air bubble, was advanced into the clear tubing and on into the Venflon by means of the saline syringe. This bolus was then injected rapidly into the antecubital vein. This method gave a good non-fractionated bolus in most patients.

The time-activity curves from both systems were analysed by the same method. A time-activity curve from the superior venacaval region of interest was used to check that the bolus was not fractionated. The gamma camera was used to image the superior vena cava and a region of interest over the right lung was entered visually, whereas with the nuclear probes this information was taken directly from the detectors. If the time-activity curve from a region of interest over the right lung was acceptable it was analysed by gamma variate fits. ${ }^{23}$ Where the bolus was attenuated we used deconvolution analysis ${ }^{5-7}$ to correct the lung time-activity curve before the shunt was calculated.

We used the same software (Gamma 11) for gamma variate fitting for data from both the gamma camera and the nuclear probe, which were both written to a file on disc in the same format.

\section{OXIMETRIC TECHNIQUE}

During right heart catheterisation paired blood samples were taken from the inferior vena cava, low, mid, and high right atrium, superior vena cava, apex, mid, portion, and outflow of the right ventricle, and main pulmonary artery. An arterial sample was obtained from a femoral needle puncture. The samples were $\stackrel{D}{\mathcal{C}}$
taken in rapid sequence once the patient was con- $c$ sidered to be physiologically stable. The oxygen $\ddot{\vec{F}}$ saturation was measured with a reflectance $\stackrel{5}{9}$ oximeter. ${ }^{89}$ The pulmonary to systemic flow was? calculated by the formula:

$\frac{\mathrm{QP}}{\mathrm{QS}}=\frac{\text { systemic arterial } \mathrm{So}_{2}-\text { mixed venous } \mathrm{So}_{2}}{\text { pulmonary venous } \mathrm{So}_{2}-\text { pulmonary arterial } \mathrm{So}_{2}}$

where $\mathrm{SO}_{2}$ is the oxygen saturation. The mixed venous oxygen saturation was estimated from saturation in the superior vena cava (SVC) and inferior vena cava (IVC) by the formula ${ }^{10}$ :

Mixed venous $\mathrm{So}_{2}=\frac{3 \times \mathrm{SVC} \mathrm{So}_{2}+\mathrm{IVC} \mathrm{So}_{2}}{4}$

DATA ANALYSIS
The calculated pulmonary to systemic flow ratios calculated by both techniques were compared by $\vec{D}$ constructing scatter plots and calculating the standard deviation about the mean difference. Correlation coefficients were not calculated because $\oplus$ according to Altman and Bland they are not appropriate in method comparison studies. ${ }^{11}$ Only 0 the lines of identity are shown in the figures. The important question is how well the two measures agree? We calculated the difference between the two measurements for each subject. The mean of these $\mathscr{Q}$ differences is a measure of the accuracy (bias) and the $\overrightarrow{\overrightarrow{0}}$ standard deviation is a measure of precision. The size 3 of the shunt was graded arbitrarily: $1=$ no shunt; 2 F $=\mathrm{QP}: \mathrm{QS}$ from $1 \cdot 3: 1$ to $1 \cdot 5: 1 ; 3=\mathrm{QP}: \mathrm{QS}$ from 1.6:1 to $2: 1$; and $4=>2: 1$.

To compare shunt size as graded by the radiologists and by the nuclear techniques the arbitrary grading was transformed to that used by the radiologists. This change was used solely to compare the shunt sizes as graded by the radiologists with the sizes given by nuclear techniques.

\section{Results}

Thirty four patients were recruited into the study. ON Four data sets were rejected-three because of failure $N$ of the nuclear probe and another patient's data were $\underset{\mathrm{W}}{N}$ lost when the storage disc was corrupted and one patient's data lost from the gamma camera because of ${ }_{0}$ technical problems. This left 29 data sets for compar- $\frac{\overparen{\Phi}}{\mathscr{D}}$ ison of the nuclear probe and gamma camera and $30 \stackrel{\mathcal{P}}{?}$ data sets for the comparison of the nuclear probe and 0 radiologists.

The size of the shunt as estimated by the nuclear probe and gamma camera showed complete accord in $\stackrel{\mathbb{Q}}{\Omega}$ 24 patients and discord in five patients (table 1). When the nuclear probe was compared with 
Table 1 Concordance of size of shunt

Complete agreement

$\begin{array}{rrr}24 & 5 & - \\ 11 & 4 & - \\ 8 & 6 & - \\ 11 & 16 & 3 \\ 14 & 13 & 4 \\ 17 & 9 & 4\end{array}$

Disagreement (by 1 grade)
Gamma camera vs nuclear probe

Nuclear probe vs oximetry

Gamma camera vs oximetry

Nuclear probe $v$ s first radiologist

Nuclear probe $v s$ second radiologist

Radiologist 1 vs radiologist 2
Disagreement (by 2 grades)

One set of data from the gamma camera was lost. This set came from one of the patients who underwent right heart catheterisation with oximetry.

oximetry there was complete concordance in 11 patients and minor variation in four patients (table 1 ). For the comparison of the gamma camera and oximetry the figures were eight and six respectively. There was some discordence between each radiologist but there was greater discordence between each radiologist and the nuclear probe (table 1 ).

The individual values for agreement for various comparisons are shown in the scatter plots and frequency histograms (figs 1-3). Table 2 shows the mean of differences between the methods and their respective standard deviations.
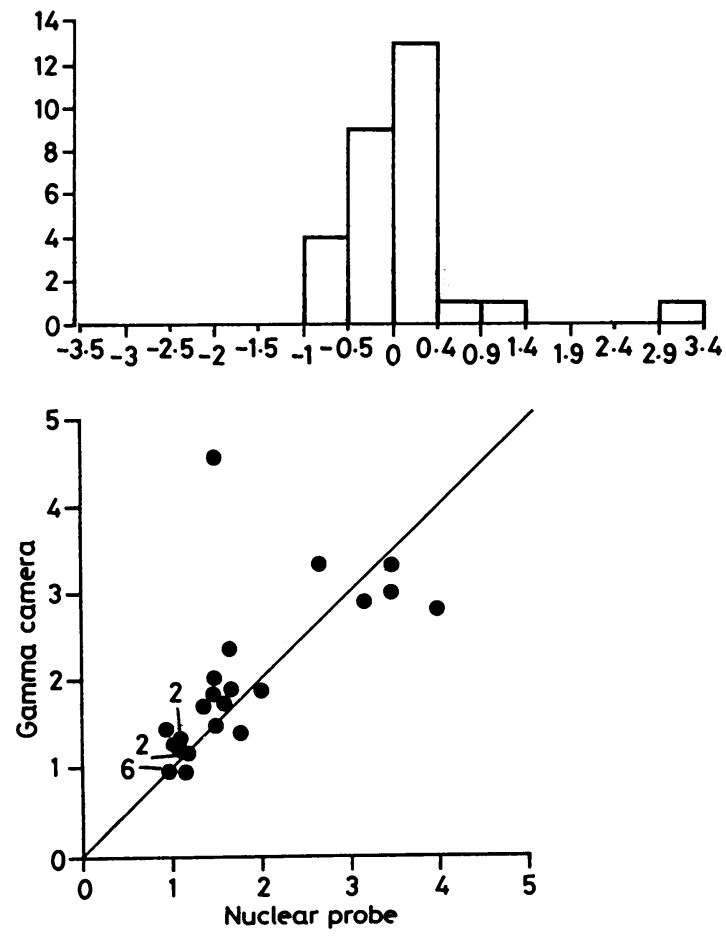

Fig 1 Scatter plot and frequency histogram of the nuclear probe and gamma camera. (The line drawn is the line of identity.) The histogram represents the differences in shunt size between the two methods. The figures on the $x$ and $y$ axes indicate the shunt size. Where data points overlap by one or more points on the scatter plot the numbers of points are given.
Generally there was a good agreement between the nuclear probe and standard gamma camera and between each isotopic method and oximetry. The larger the size of the shunt the greater the scatter of the data. This does not, however, affect clinical interpretation of the size of the shunt.

\section{Discussion}

The results reported in this study confirm the close agreement in the size of left to right shunts measured by the standard gamma camera and by the oximetric method. They also demonstrate that the cadmium telluride miniaturised nuclear probe provides estimates of shunt size that agree with the gamma camera and with the oximetric methods. During the
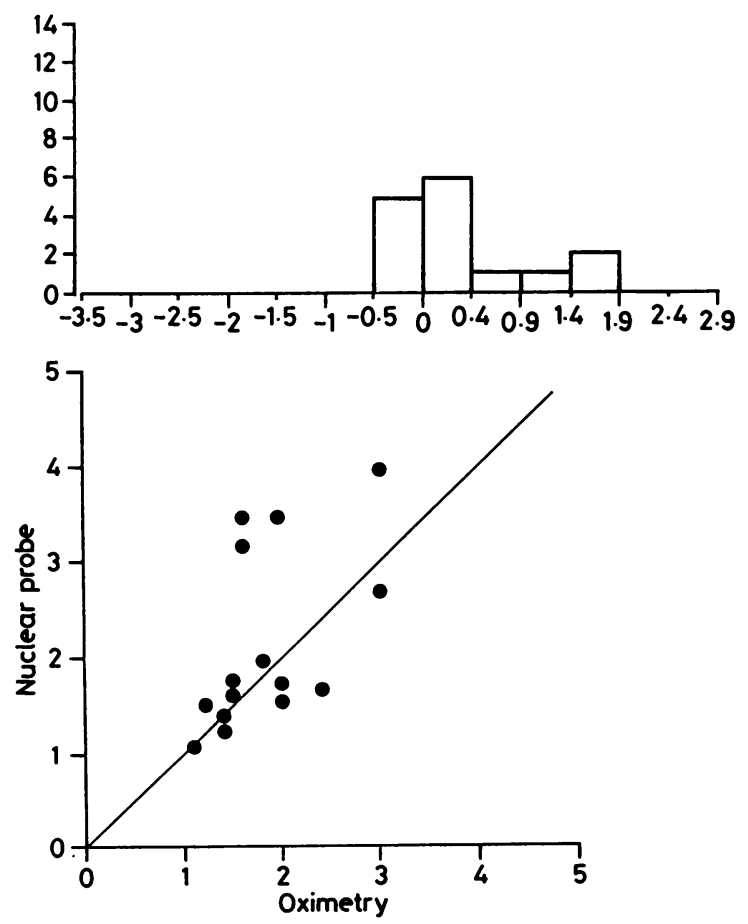

Fig 2 Scatter plot and frequency histogram of oximetry and the nuclear probe. (Details of layout as for fig 1.) 
466
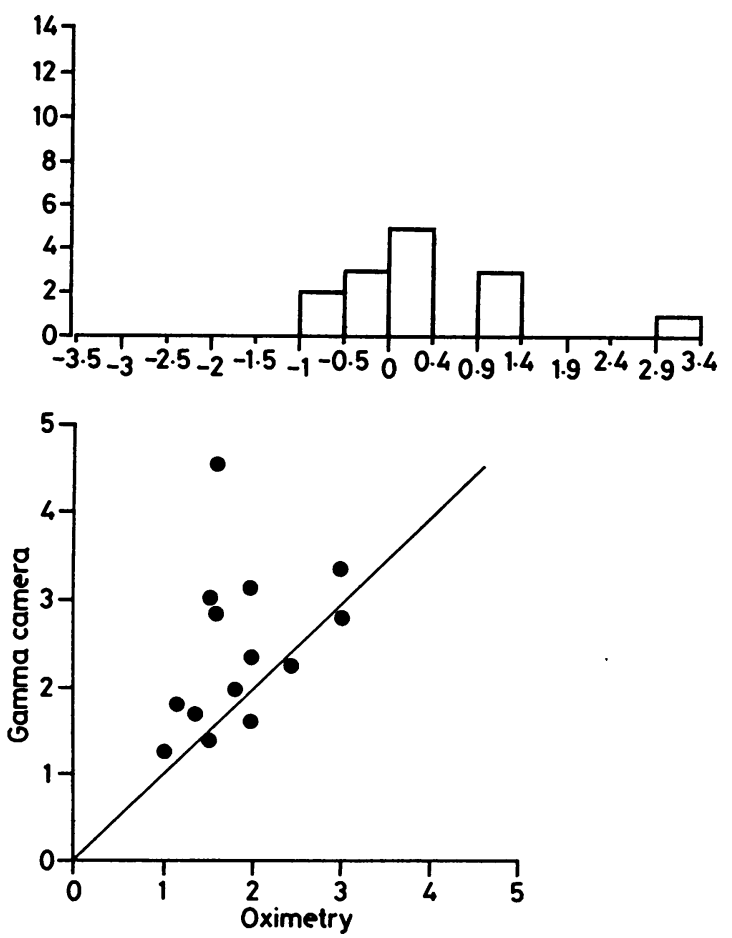

Fig 3 Scatter plot and frequency histogram of oximetry and the gamma camera. (Details of layout as for fig 1.)

study there were a few minor changes to the technique. The injection of the bolus was improved as was collimation of the cadmium telluride crystal. These changes assisted processing by producing better curves but did not affect the final results. The size of the differences between the nuclear data and oximetric data was similar at the beginning and end of the study as were the differences between the nuclear probe and gamma camera. The nuclear probe, when used alone, needs only a quarter of the dose of radiopharmaceutical required for the gamma camera.

It was instructive to note the variation in shunt size as estimated by two experienced radiologists. We conclude that radiology is therefore at best a very

Table 2 Mean (SD) shunt size for between-method comparisons

\begin{tabular}{lll}
\hline & $\begin{array}{l}\text { Mean } \\
\text { difference }\end{array}$ & $\begin{array}{l}\text { SD of } \\
\text { difference }\end{array}$ \\
\hline $\begin{array}{l}\text { Nuclear probe vs gamma camera } \\
(\mathrm{n}=29)\end{array}$ & 0.08 & 0.67 \\
$\begin{array}{l}\text { Nuclear probe vs oximetry } \\
(\mathrm{n}=15)\end{array}$ & 0.36 & 0.78 \\
$\begin{array}{c}\text { Gamma camera vs oximetry } \\
(\mathrm{n}=14)\end{array}$ & 0.53 & 0.95 \\
\hline
\end{tabular}

Gould, Turner, Keeling, Ring, Cox, Marshall $\frac{\text { D }}{\frac{T}{D}}$ rough guide to the clinical significance of the shunt $\stackrel{\varrho}{\frac{1}{c}}$ size.

The cadmium telluride miniature probe can be $\vec{\Rightarrow}$ used to detect left to right shunts and to estimate the size of the shunt. In most patients the results of the $\frac{C}{O}$ nuclear probe and gamma camera methods agreed on $\overline{\bar{c}}$. the size of the shunt (large, moderate, or small). The $\vec{\Phi}$ nuclear probe can therefore be regarded as a useful $\cong$ clinical tool. The nuclear technique becomes less $\omega$ accurate in three situations. It cannot be used to $\overrightarrow{0}$ measure right to left shunts because it works on the $\vec{\overrightarrow{ }}$ principle of measuring the increased pulmonary $\bar{\sigma}$ transit time which does not occur in this situation. The time-activity curves, from which the shunt is of calculated, may also be prolonged in severe left 0 ventricular dysfunction and in pronounced mitral valve regurgitation, making estimation of the shunt $\stackrel{\mathscr{\omega}}{\mathrm{C}}$ size unreliable; however, we found no evidence of 0 this effect in our data.

In many units the demand for the gamma camera is high. The cadmium telluride miniature detectors can provide useful data at a much lower cost than the $\vec{c}$ gamma camera, and although no pictures are avail- $\infty$ able the miniature detectors are easily portable and may be interfaced with a small microcomputer. This technique can be used in both the coronary care unit and the outpatient department. The nuclear technique complements cross sectional echocardiography, which will determine the site of the shunt. Ginzton et al showed that the combination of contrast cross sectional echocardiography and radionuclide shunt detection led to a proper diagnostic and therapeutic decision in 24 of 25 patients. ${ }^{12}$

We consider that the nuclear probe is a useful $\vec{ه}$ investigative tool in the determination of the pulmonary systemic flow ratio in patients with left to right intracardiac shunts.

\section{References}

1 Askenazi J, Ahnberg DS, Korngold E, La Farge C, Maltz DL, Treves S. Quantitative radionuclide angiography: detection and quantitation of left to $\mathrm{N}$ right shunts. Am J Cardiol 1976;37:382-7.

2 Anderson PAW, Jones RH, Sabiston DC. Quantitation of left to right cardiac shunts with radionuclide angiography. Circulation 1974;49:512-6.

3 Hurwitz RA, Treves S, Keane JF, Girod DA, Caldwell RL. Current value of radionuclide angiocardiography for shunt quantification and management in patients? with secundum ASD. Am Heart J 1982;103:421-5.

4 Baker EJ, Ellam SV, Lorber A, Jones ODH, Tynan MJ, Maisey MN. Superiority of radionuclide over oximetric measurement of left to right shunts. $\mathrm{Br}$ Heart $J$ 1985;53:535-40.

5 Alderson PO, Jost RG, Straus AW, Boonvisut S, ? Markham MS. Radionuclide angiography. Improved 
diagnosis and quantitation of left to right shunts using area ratio techniques in children. Circulation 1975;51:1136-43.

6 Alderson PO, Douglass KH, Mendenhall KG, et al. Deconvolution analysis in radionuclide quantitation of left to right cardiac shunts. $J$ Nucl Med 1978;20:502-6.

7 Ellam SV, Wright JGC, Maisey MN. Pitfalls on using deconvolution in the analysis of left to right shunts using first pass radiography. Nucl Med Commun 1983;4:35-9.

8 Holling HE, Macdonald I, O'Halloran JA, Venner A. Reliability of a spectrophotometric method of estimating blood oxygen. J Appl Physiol 1955;8:
249-54.

9 Polani ML, Mehir RM. New reflectance oximeter. Rev Sci Instrum 1960;31:401-3.

10 Miller HC, Brown DJ, Miller GAH. Comparison of formulae used to estimate oxygen saturation of mixed venous blood from caval samples. $\mathrm{Br}$ Heart $J$ 1974; 36:446-51.

11 Altman DG, Bland JM. Measurement in medicine. The analysis in method comparison studies. Statistician 1983;32:307-17.

12 Ginzton LE, French W, Mena I. Combined echocardiographic and radionuclide diagnosis of ASD: accuracy of the technique and analysis of erroneous diagnoses. Am J Cardiol 1984;53:1639-42. 